\title{
La consolidación del aprendizaje-servicio en Fundesplai y en la educación en el tiempo libre
}

\author{
Carles Xifra Cirach y Susagna Escardíbul Tejeira \\ Fundesplai
}

\section{Resumen}

A través de este artículo revisamos el proceso de generalización del aprendizajeservicio que ha tenido lugar en Fundesplai y en el ámbito de la educación en el tiempo libre. Son diversas las estrategias y los mecanismos que nos han llevado, en poco más de 15 años, del descubrimiento a la consolidación del aprendizaje-servicio en la cultura de la organización. Fundesplai, a través de su acción centrada en el impulso de la educación en el tiempo libre con voluntad transformadora, plantea que el aprendizaje-servicio representa la integración de dos intencionalidades que considera básicas para la organización. Por una parte, el fomento del trabajo por competencias y habilidades entre las chicas y los chicos y, por otra parte, el compromiso social como finalidad de los programas socioeducativos que lleva a cabo. La incorporación del aprendizaje-servicio en la entidad está contribuyendo a formar personas competentes pero, sobre todo, personas capaces de asumir retos, de reflexionar críticamente y de actuar en su entorno, buscando, de forma altruista, un impacto positivo en sus comunidades.

\section{Palabras clave}

Educación en el tiempo libre, aprendizaje-servicio, metodología del proyecto, formación de equipos, programa pedagógico. 


\title{
The consolidation of service-learning in Fundesplai and in free-time education
}

\begin{abstract}
This article reviews the expansion of service-learning at Fundesplai (The Catalan Leisure Time Foundation) and in the wider context of informal education. Over the past 15 years or so, multiple strategies and mechanisms have guided us from initial implementation to the consolidation of service-learning within the organisation. Through actions focusing on promoting education during leisure time with a transformational will, Fundesplai proposes that service-learning represents the integration of two aims that are central to the foundation: firstly, the promotion of competency and skill-based work for young people, and secondly, placing social engagement at the core of the socio-educational programmes that the foundation carries out. The introduction of service-learning in the foundation is helping to train young people who will not only be competent but who will also be able to take on new challenges, reflect critically and take action in their environment, seeking, altruistically, to achieve a positive impact on their communities.
\end{abstract}

\section{Keywords}

Free-time education, service-learning, project methodology, team building, pedagogical program. 


\section{Introducción}

En el año 2003, Fundesplai compartió con Alberto Croce, director entre los años 1999 y 2015 de la Fundación SES, de Buenos Aires, un seminario sobre experiencias educativas en Argentina y Latinoamérica fundamentadas en el aprendizaje-servicio. Estas experiencias conectaban a su vez con la extendida corriente del Service Learning en Norteamérica. Aquel seminario y el conjunto de reflexiones que surgieron nos asombraron de tal manera que fue una semilla que en poco tiempo germinaría y crecería, extendiendo el aprendizaje-servicio en todo el territorio catalán y, en particular, en nuestra organización y en el ámbito de la educación en el tiempo libre.

Fundesplai es una entidad de la sociedad civil, sin ánimo de lucro que, desde hace más de 45 años, impulsa la educación en el tiempo libre de las niñas, niños y jóvenes y trabaja para favorecer el desarrollo de organizaciones de base infantiles y juveniles de educación en el tiempo libre. Además, fomenta el fortalecimiento de las asociaciones del Tercer Sector y promueve la ciudadanía y la inclusión social con voluntad transformadora. Fundesplai cuenta con un amplio abanico de programas y servicios educativos y sociales que se concretan en actividades y acciones en el ámbito de la educación en el tiempo libre (actividades extraescolares, campamentos de verano, comedores escolares...); programas de educación ambiental; programas de promoción de la salud y habilidades para la vida; programas de alfabetización digital; programas formativos, de orientación y de inclusión socio laboral, entre otros. Actualmente, Fundesplai es uno de los principales movimientos de educación en el tiempo libre en Catalunya y agrupa la acción de una Federación que cuenta con 115 centros y proyectos de educación de tiempo libre (clubs infantiles y juveniles, proyectos de inclusión de personas con diversidad funcional, acción intergeneracional y socio comunitaria, etc.). En global moviliza a 1.494 monitores y monitoras y a 16.119 niños y niñas y jóvenes (Informe Fundesplai, 2017-2018).

En poco tiempo, el aprendizaje-servicio se ha extendido como una gota de aceite entre las entidades de educación en el tiempo libre en Catalunya $y$, especialmente, en Fundesplai. Son diversos los factores que explican este rapidísimo contagio, pero uno de los más determinantes ha sido que la educación en el tiempo libre y la acción de los centros se fundamentaba en una concepción, en una mirada y en una metodología educativa muy similar a la propuesta del aprendizaje-servicio, aunque no la nombrasen de la misma forma.

La educación en el tiempo libre es esencialmente educación en valores y responde a un modelo educativo en el cual se activan los principios de la experiencia, el altruismo, la participación, la cooperación y la ciudadanía. Puesto que el ApS comparte estos principios, reconocerlos supuso el descubrimiento de un "método innovador, un concepto nuevo y más original" (Puig et al., 2006, 12), y representaba una práctica que no solo proponía nuevos retos, sino

Xifra, C.; Escardíbul, S. (2018). La consolidación del aprendizaje-servicio en Fundesplai y en la educación en el tiempo libre. RIDAS, Revista Iberoamericana de Aprendizaje Servicio, 6, 115-126. 
también mejoras substanciales a los proyectos sociales y educativos en general.

Este factor, conjuntamente con otros aspectos que se analizan a continuación, explican el porqué y el cómo el aprendizaje-servicio pasa de un contagio inicial a interiorizarse e institucionalizarse en la cultura de Fundesplai. Por destacar algunos datos cuantitativos, en el año 2017, Fundesplai, y los centros de educación en el tiempo libre que agrupa, desarrolló un conjunto de 59 proyectos de aprendizaje-servicio, donde participaron más de 1.200 niñas, niños y jóvenes. De este conjunto diversos de proyectos, el $46 \%$ corresponden al ámbito de la ayuda próxima, el 32\% al ámbito del medioambiente y el $22 \%$ al ámbito de la participación ciudadana. Este mismo año se han titulado 1.381 monitoras y monitores (formación reglada en educación en tiempo libre) los cuales han recibido formación específica en aprendizaje-servicio.

\section{Del contagio a la institucionalización}

Los molinos de viento producen energía eólica gracias al movimiento circular de sus aspas. Esta puede ser una buena analogía para explicar el proceso de institucionalización del ApS en Fundesplai y en la educación en el tiempo libre en general.
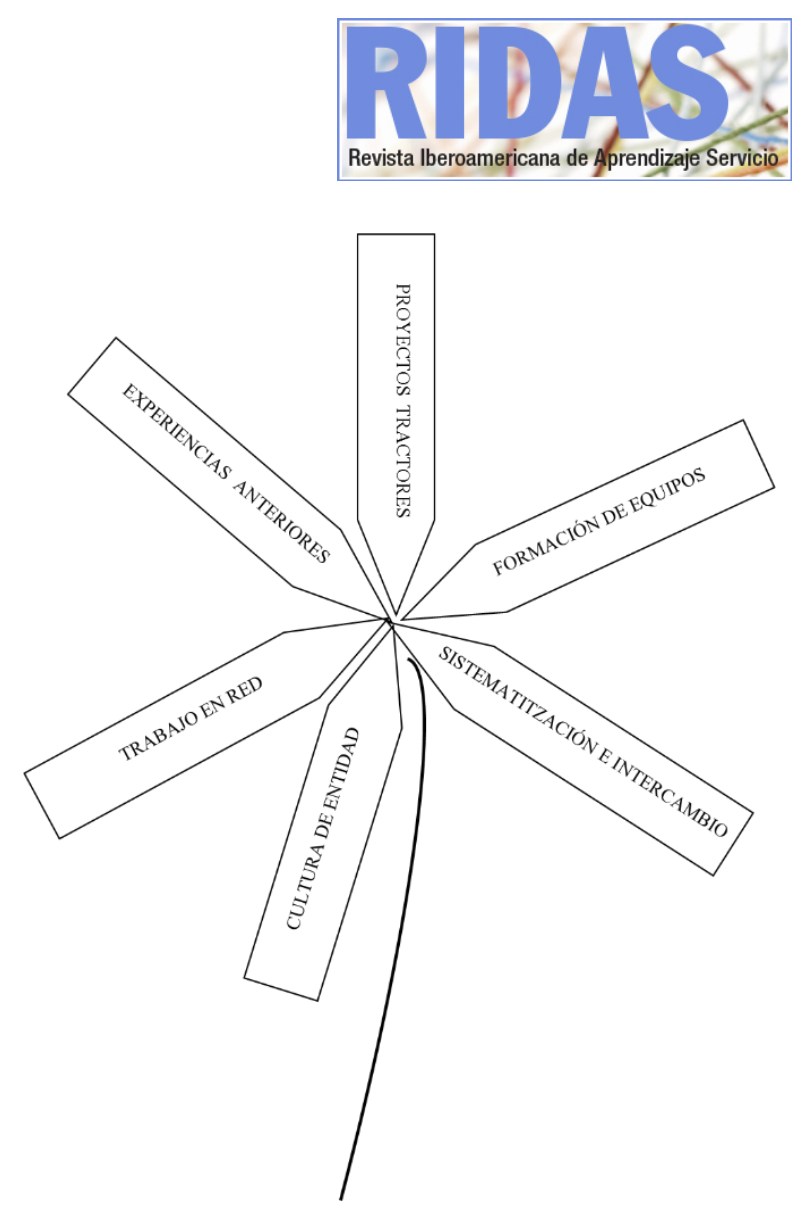

\section{Reconocimiento de experiencias pedagógicas anteriores}

Analizando este tema con perspectiva, es necesario reconocer que Fundesplai, y la educación en el tiempo libre en general, ya disponía de un terreno abonado y preparado para la siembra del aprendizaje-servicio. La buena acogida inicial se debió, en parte, a que no reconocimos el ApS como un invento educativo nuevo, sino que dio nombre y bases conceptuales y metodológicas muy potentes a muchas de las prácticas que llevábamos cabo en el ámbito educativo del tiempo libre desde hacía muchos años.

Además, el ApS encajaba con la pedagogía del proyecto y los centros de interés en que se basa la educación en el tiempo libre. El trabajo por proyectos se consolidó en este ámbito gracias a 
las aportaciones de diversas corrientes pedagógicas de la mano, entre otros, de Dewey, Kilpatrick, Decroly, Makarenko y Freire. $Y$ en el ámbito más específico de la educación en el tiempo libre, autores como Baden Powell, Joaquim Franch y Alfons Martinell, pedagogías que comparten buena parte de los principios educativos del ApS:

- La proyección social del proyecto y la voluntad inicial de contribuir al bien común, a la mejora del entorno y la comunidad

- La participación y el protagonismo de las chicas y los chicos en la detección de necesidades, diseño y desarrollo de la propuesta

- La experiencia de intervención real como motor principal de aprendizaje (Dewey, 1916)

- La significación y la conexión con las inquietudes y los intereses de los y las participantes

- La adquisición de valores a través de la participación en actividades de compromiso cívico

Así pues, Fundesplai partía de experiencias consolidadas cercanas al aprendizaje-servicio que trabajaban la capacidad para pensar alternativas, resolver creativamente problemas y que, además, también favorecían la implicación de las niñas y los niños aumentando su confianza. Ante todo, contábamos con la predisposición de un buen número de entidades de educación en tiempo libre atentas a las necesidades del entorno y con bagaje para articular proyectos de transformación. Se trataba, por tanto, de aprovechar el trabajo por proyectos y envolverlo con la potente idea de ponerlo al servicio de la comunidad.

El aprendizaje-servicio era una innovación que encajaba con la larga tradición de experiencias solidarias previas que había desarrollado Fundesplai a través de diversos proyectos, tales como el proyecto Todos Somos Meninos o los Campos de Trabajo de Verano dirigidos al colectivo de jóvenes. La cultura de la solidaridad es uno de los valores sobre los cuales se erige la acción de Fundesplai, incorporando el "pensar y actuar por los otros", y optando por la cultura de la cooperación, basada en la dignidad de las personas y de los pueblos, y en el respeto a la diversidad y a los Derechos Humanos (Carrasco, 2015).

En el año 1994 se gestó el proyecto de solidaridad Todos Somos Meninos que hermanaba Fundesplai con el Movimiento Nacional de Meninos e Meninas de Rua de Brasil. El eje vertebrador del proyecto fueron los viajes de grupos de jóvenes a Brasil y su participación en el Encontro Nacional de Meninos e Meninas de Rua. Las posibilidades de trabajo aumentaban cuando los grupos regresaban y, además de compartir la experiencia, se comprometían en acciones de difusión y denuncia de la injusticia social en la que vivían miles de niños y niñas en las calles de las grandes ciudades brasileras y la lucha por la defensa de sus derechos (Rubio y Escardíbul, 2010).

El verano del 1994, Catalunya vivió una serie de incendios devastadores que afectaron a muchas zonas forestales

Xifra, C.; Escardíbul, S. (2018). La consolidación del aprendizaje-servicio en Fundesplai y en la educación en el tiempo libre. RIDAS, Revista Iberoamericana de Aprendizaje Servicio, 6, 115-126. 
del país. Fundesplai quiso ayudar y, con el acuerdo del Departamento de Juventud del gobierno catalán, organizó diversos campos de trabajo para jóvenes que fueron pioneros y referentes de actuaciones posteriores. Jóvenes llegados de todo el país dedicaban en la época estival tiempo y energías para recuperar una zona natural y quemada a la vez que conocían el bosque mediterráneo y tomaban conciencia de los riesgos, las consecuencias y las medidas de prevención de los incendios forestales. Desde aquel año Fundesplai continúa organizando, verano tras verano, distintos campos de trabajo de mejora del entorno natural.

Encontramos otros ejemplos de proyectos solidarios muy significativos, como el intercambio con jóvenes de Bosnia después del conflicto armado, o los grupos de jóvenes que viajaron a Galicia el verano del 2002 para ayudar a retirar el chapapote del barco Prestige $y$, de ese modo, colaborar para reducir el gravísimo impacto ambiental del vertimiento de petróleo en las costas gallegas.

Y para acabar de trabar la propuesta del aprendizaje-servicio con todo aquello que ya estábamos haciendo, destacamos como el ApS concuerda con la visión y sentido de la educación. En efecto, la educación en el tiempo libre es, sobre todo, educación en valores orientada a desplegarlos a través de la acción y de la experiencia. Es decir, los valores se aprenden practicándolos colectivamente. Y desde Fundesplai hemos considerado siempre que la educación en valores debe orientarse al compromiso social de las niñas, los niños y los jóvenes. Y desde esta mirada, los centros de educación en el tiempo libre se convierten en pequeñas escuelas de democracia y ciudadanía, donde se fomenta el protagonismo juvenil, el sentimiento de pertenencia a sus comunidades, la participación y la corresponsabilización en los asuntos colectivos y el deseo de convivir en una sociedad más justa y solidaria. Planteamiento estrechamente compartido con la intencionalidad educativa del aprendizaje servicio, "pensado para formar ciudadanía capaz de participar en la búsqueda del bien común" (Puig et al., 2006, 37).

\section{Impulso de proyectos tractores}

Más allá de la buena acogida inicial y de todas las experiencias previas solidarias, existe otro elemento importante que explica la rápida expansión del aprendizaje-servicio en nuestra organización: el impulso de la innovación educativa a través de proyectos que nombramos intencionalmente proyectos tractores. Los proyectos tractores son iniciativas que, por sus características, promueven un cambio metodológico y se convierten en modelos referentes que pueden ser replicables y escalables.

Reconocemos tres proyectos tractores que fueron clave para consolidar y divulgar el aprendizaje-servicio en Fundesplai, pero también para el resto de agentes educativos y sociales vinculados a la organización: el proyecto de Donación de Sangre y Educación para la Ciudadanía, el 
proyecto Conecta Joven y el proyecto Jóvenes por el Barrio.

Hacia el año 2005, con el proyecto de Donación de Sangre y Educación para la Ciudadanía iniciábamos formalmente el camino para impulsar las experiencias educativas basadas en el ApS. Muchos centros de educación en el tiempo libre implicaron a niños, niñas y jóvenes en la tarea de difundir la necesidad social de garantizar e incrementar las donaciones de sangre a la vez que descubrían, desde otra perspectiva, contenidos relativos a la sangre y a la promoción de la salud. El proyecto fue un estímulo en muchos sentidos: supuso un entrenamiento en el diseño de proyectos con metodología ApS; sirvió para explicitar contenidos y conocimientos instrumentales, una cuestión a veces ignorada en el ámbito educativo del tiempo libre; y amplificó el trabajo en red ya que implicó la colaboración de distintas y diversas entidades (Banco de Sangre y Tejidos, Universidad, centros de salud local, etc.). Cabe destacar que en los primeros años del proyecto contamos con el apoyo del grupo de investigación en Educación Moral de la Universidad de Barcelona. Su orientación, tanto en la ejecución, como en la sistematización de la experiencia fue clave para la consolidación del proyecto en los centros educativos de tiempo libre.

Casi simultáneamente se impulsó el proyecto Conecta Joven. Implicó a grupos de jóvenes que se formaron en el uso de las tecnologías de la información y de la comunicación y en habilidades comunicativas para ayudar a personas mayores y a otros colectivos a superar la brecha digital, debido a la falta de formación o a la imposibilidad de acceder a procesos de aprendizaje sobre el uso de estas herramientas. Se trata de uno de los primeros proyectos marcadamente intergeneracionales y de ayuda próxima de gran impacto y alcance, ya que la iniciativa se extendió a todo el territorio español. Con este proyecto también conseguimos fortalecer el vínculo educación formal-educación no formal, puesto que las jóvenes y los jóvenes participantes provienen mayoritariamente de centros educativos.

Por último, el proyecto Jóvenes por el Barrio (JxB). Se trata de una experiencia a través de la cual grupos de jóvenes reciben una formación básica sobre educación en el tiempo libre y, posteriormente, colaboran de forma activa con los centros, asumiendo la función de ayudantes de monitor/a. Con este proyecto las entidades de tiempo libre son, por primera vez, receptoras de un servicio que se orienta a cubrir las necesidades sociales identificadas. Es una propuesta que refuerza el trabajo comunitario en los barrios e integra también la acción de los centros educativos. Este proyecto ha sido motor de muchas otras iniciativas parecidas. Asimismo, ha contribuido significativamente a mejorar el éxito educativo de los y las participantes, de modo que ha corroborado muchas de las tesis que ponen de manifiesto que el ApS impacta positivamente en los y las estudiantes (Furco, 2005). 
Fundesplai dotó, acertadamente, de una dimensión colectiva a estos proyectos. Muchos niños, niñas y jóvenes de diferentes entidades de tiempo libre los estaban impulsando paralelamente en diferentes territorios y barrios, lo que nos permitió sistematizar procesos, identificar etapas y fases, profundizar sobre el sentido del proyecto e incorporar rápidamente mejoras a través de evaluaciones continuas. Además, nos brindó la oportunidad de compartir el impacto social, educativo, mediático, y comunitario de tales experiencias, hecho que motivó la implicación y participación de otras entidades.

\section{Sistematización e intercambio de buenas prácticas}

De manera análoga a los proyectos tractores, la consolidación del aprendizaje-servicio se explica, también, por la constante y ardua tarea de sistematización y divulgación de prácticas exitosas.

En primer lugar, la voluntad de sistematizar cada una de las experiencias y de presentarlas utilizando diferentes formatos (documentos, blogs, material audiovisual, informes, etc.), de recoger los dinamismos básicos, las dificultades y los aciertos, y de hacerlo con la participación directa de los equipos educativos responsables, se convierte en un proceso de reflexión y de aprendizaje continuo muy enriquecedor. Uno de los dinamismos en el cual hemos profundizado más es en la importancia del reconocimiento público y la celebración del cierre del proyecto. (Puig y Bär, 2016).

Seguramente siempre, pero especialmente en el ámbito de la educación en el tiempo libre, donde el voluntariado y la motivación por la implicación en los temas colectivos es esencial, la etapa del reconocimiento al servicio realizado toma una dimensión que va mucho más allá de la interiorización de los aprendizajes y de impactar en la autoestima de los y las participantes y en la construcción de un auto concepto positivo (Cerda, 2015). Influye positivamente en los procesos de empoderamiento y en la capacidad para movilizarse e implicarse en nuevos retos.

En segundo lugar, la difusión de buenas prácticas se ha articulado a menudo a través de la organización de jornadas de intercambio. Destacamos en positivo y como experiencia a replicar, la realización de encuentros mixtos donde adultos, niñas y niños dialogan y comparten propuestas, así como encuentros entre iguales, donde jóvenes de todas las edades se reúnen para socializar sus proyectos y debatir sobre el sentido de su participación. Para nosotros fue especialmente interesante la iniciativa Foro Somos Niños y Niñas, Somos Presente, iniciado el año 2015, una jornada liderada por los niños y planeada como espacio de metaparticipación (Trilla y Novella, 2001), donde repensar el valor de la participación social y visibilizar públicamente sus iniciativas de aprendizaje-servicio. 


\section{Formación de equipos}

La formación de monitores y monitoras entorno al aprendizaje-servicio ha sido intensa y continua y, sin lugar a dudas, ha sido fundamental para extender la metodología en la organización. Fundesplai dispone de diferentes espacios formativos donde participan un gran número de educadoras y educadores de los centros de educación en el tiempo libre. En estos espacios se organizan de forma reiterada, formaciones de iniciación al aprendizaje-servicio, cápsulas sobre temas específicos (participación, rúbrica para la evaluación, etc), así como seminarios para profundizar en los diferentes elementos que caracterizan el ApS. Se trata de formaciones de diferentes formatos, intensidades y duración, que han permitido llegar a todos los niveles de responsabilidad de los equipos educativos y de los programas de educación en el tiempo libre.

El centro de formación de Fundesplai está homologado para impartir la formación oficial reconocida por el gobierno de Catalunya de Dinamización de Actividades de Educación en el Tiempo Libre Infantil y Juvenil (150 h. teóricas y $160 \mathrm{~h}$. de prácticas) y de Dirección de Actividades de Educación en el Tiempo Libre Infantil y Juvenil (200 h. teóricas y $120 \mathrm{~h}$. prácticas). Son titulaciones requeridas legalmente para la realización de gran parte de las actividades de educación en el tiempo libre en Cataunya. En ambas formaciones se ha incorporado el ApS como contenido curricular. Con ello hemos conseguido difundir de forma sistemática la metodología y formar en la ética de la solidaridad a los futuros monitores y monitoras. El volumen de participantes en estas formaciones es significativo, solo en el año 2017 el centro de formación de Fundesplai tituló a 1.381 personas.

\section{Tejer redes}

Y no hacerlo solos. Como subraya Uruñuela (2016:10) "no se trata solamente de garantizar la coordinación entre todas las entidades, sino de llegar a un trabajo en red basado en la cooperación". La red y el entramado de conocimientos y experiencias que se compartieron a través de las organizaciones impulsoras del Centro Promotor de Aprendizaje Servicio en Catalunya fue, y es, una fuente de inspiración, de enriquecimiento y de motivación constante. Impulsamos el ApS conjuntamente con otros movimientos de educación en el tiempo libre y con la comunidad educativa pero también, y sobre todo, con entidades sociales y ambientales que desde el ámbito del Tercer Sector se vinculaban a la propuesta.

En el trabajo en red, cada entidad, organismo o persona establece sus conexiones $y$, con el paso del tiempo, la estructura evoluciona hacia un sistema de cooperación más maduro, donde todos son indispensables. En este proceso reconocemos como actor clave al grupo de trabajo del Instituto de Ciencias de la Educación de la Universidad de Barcelona. Tuvo un papel destacado en la capacitación de las personas que lideraban la implementación del aprendizaje- 
servicio en sus entidades de referencia, a la vez que se comprometían con el diseño de formaciones iniciales y avanzadas para impulsarlas en cascada en diferentes ámbitos y territorios.

Por otro lado, desde el año 2012 colaboramos con la realización de acciones formativas inscritas en el programa de aprendizaje-servicio de la Universidad de Barcelona y más recientemente hemos iniciado el mismo camino con la Universidad Internacional de Catalunya. Estas experiencias no solo acercan el alumnado universitario a la tarea social de las organizaciones, objetivo en sí mismo suficientemente importante, sino que también generan oportunidades de reconocimiento, de vínculo y de creación de conocimiento compartido. Un buen ejemplo de las complicidades y las sinergias que surgen es la investigación que está llevando a cabo la Universidad Internacional de Catalunya con el título "Impacto de la participación interna que generan los proyectos de aprendizaje servicio en Fundesplai", en desarrollo a fecha de hoy. Con seguridad, esta investigación nos reportará evidencias sobre la incidencia de los proyectos ApS en el ámbito de la educación en el tiempo libre, un ámbito en el que, además, es muy necesario promover la investigación científica.

\section{Institucionalización y cultura de la entidad}

Y, para acabar, nos gustaría reflexionar un poco más sobre el efecto que produce el cambio de mirada que propone el aprendizaje-servicio dentro de la organización. Su impulso a través de las acciones formativas, de reconocimiento y de la socialización de buenas prácticas, de la publicación de artículos, etc., contribuyen a hacer realidad una implementación transversal del ApS en muchos de los programas sociales y educativos de Fundesplai. Progresivamente el aprendizaje-servicio se ha incorporado en los programas de promoción de la salud y de educación ambiental, en los espacios educativos de los comedores escolares, en los programas dirigidos a jóvenes en situación de riesgo social, incluso en los programas más específicos dirigidos a personas privadas de libertad.

Desde hace más de 25 años, Fundesplai desarrolla un programa pedagógico de educación en valores que se dirige al conjunto de niñas, niños, jóvenes, familia y comunidad en general con la que trabaja. Estos programas son propuestas de intervención educativa de carácter lúdico, dramático, plástico, ecológico, musical, etc. que se articulan entorno a un centro de interés o proyecto global. En el año 2008, pusimos en marcha la campaña Ciudadanía Comprometida que aglutinó cuatro programas para fomentar el sentido de la corresponsabilidad de niños, niñas jóvenes con la transformación de su entorno, incorporando el aprendizaje servicio como metodología básica. Son los programas Barrinem -2008-2009propuesta educativa para el civismo y la convivencia; Mandala -2009-2010-, propuesta educativa por la interculturalidad y la fraternidad; Mirall -2010-2011-, propuesta educativa por

Xifra, C.; Escardíbul, S. (2018). La consolidación del aprendizaje-servicio en Fundesplai y en la educación en el tiempo libre. RIDAS, Revista Iberoamericana de Aprendizaje Servicio, 6, 115-126. 
la igualdad de oportunidades; y, Àgora -2011-2012-, propuesta educativa para la participación.

Y en el año 2012 se elaboró la propuesta educativa Tejiendo Ciudadanía Comprometida, centrada en la promoción y el impulso de nuevos proyectos de aprendizaje-servicio. Con esta propuesta conseguimos recopilar un banco de buenas prácticas fácilmente transferibles y logramos animar a muchos otros centros a iniciarse en el aprendizaje-servicio. Además, surgieron los proyectos ApS colectivos y compartidos, como fue la iniciativa de Proyectos de Custodia de Espacios Naturales. En estos proyectos, las y los jóvenes participan colectivamente y se comprometen con el mantenimiento o mejora de determinadas zonas naturales de valor ecológico, por ejemplo, mantener la biodiversidad de los prados de pasto de alta montaña, en retroceso debido al abandono de la actividad ganadera en las montañas de los Pirineos catalanes.

Volvamos a la analogía del molino de viento: para que las aspas se muevan con fuerza, es necesario aire fresco y renovado de tanto en tanto. Se necesitan sacudidas periódicas que actualicen el sentido del aprendizajeservicio en la educación en el tiempo libre. En este sentido Fundesplai ha impulsado nuevos proyectos entorno a la promoción de la participación ciudadana a partir de campañas de sensibilización y donde el uso de las tecnologías y las redes sociales se incorpora como instrumento central. Uno de estos proyectos es el festival de cortometrajes para la transformación social Edufilms. A través de esta propuesta, diferentes grupos de jóvenes debaten sobre una determinada temática social que les preocupa o les interesa especialmente y profundizan sobre ella contrastando puntos de vista, ampliando la información, formándose. Posteriormente preparan el guion, elaboran un plan de grabación, escogen músicas, buscan los espacios de rodaje, etc. Se trata de una actividad de aprendizaje muy potente donde se adquieren nuevos conocimientos $y$ habilidades relacionadas con el mundo del cine y que sirve para estimular el pensamiento crítico y acercarse a todo tipo de realidades. Los cortometrajes se exponen en un festival que es en sí mismo un acto de reconocimiento, donde además de compartir los trabajos realizados constituye el punto de partida de las acciones de servicio que los grupos llevaran a cabo, entre otras, charlas en los centros sociales y educativos o campañas de sensibilización a través de las redes sociales.

\section{Reflexiones finales}

Así pues, el camino del aprendizajeservicio en Fundesplai ha sido y será un largo camino de interiorización de una forma compartida de entender la educación. Un camino que ha transformado la organización y que nos ha reportado muchísimos aprendizajes.

Por una parte, la consolidación del aprendizaje-servicio en nuestra organización solo se puede entender a partir de la suma de diversas estrategias que van desde el 
reconocimiento de experiencias cercanas al impulso de proyectos tractores colectivos, a la sistematización de buenas prácticas, a acciones de divulgación, formación continua, creación de alianzas con distintos actores y organismos, etc.

Por otra parte, el aprendizaje-servicio ha contribuido a recuperar y reimpulsar prácticas y experiencias de valor con sentido social y educativo en nuestro ámbito. Esto está ayudando a transformar y mejorar la relación entre las escuelas, las entidades sociales y las ciudades, haciendo más posible la idea de comunidades y ciudades educadoras.

Hoy no podemos concebir la acción socioeducativa de Fundesplai sin la mirada del aprendizaje-servicio y esperamos que las chicas y los chicos que han crecido con los programas ApS en los últimos 15 años se movilicen, lideren y participen activamente en la construcción de un mundo mucho mejor.

\section{Referencias bibliográficas}

Cerda, M. ( 2015). Una experiència significativa incluye participación, cooperación, reflexión y reconocimiento. En: Puig, J. (coord.). 11 ideas clave ¿Cómo realitzar un proyecto de aprendizaje Servicio? (pp. 47-62). Barcelona: Graó.

Carrasco, S. (2015). Cultura, Valors $i$ Ética. Barcelona: Fundesplai.

Disponible en: https://fundesplai.org/arxius/PDFs/Publ icacions/cultura valors i etica llibre.p df
Furco, A. (2005). Impacto de los proyectos de aprendizaje-servicio. En Actas del 7mo. Seminario Internacional Aprendizaje y Servicio Solidario.

Buenos Aires: CLASYSS. (pp. 19-26). Disponible en

http://www.clayss.org.ar/seminario/an teriores/actas/2005 Actas7.pdf

Puig, J.M.; Batlle, R.; Bosch, C.; Palos, J .(2006). Aprendizaje Servicio. Educar para la Ciudadanía. Barcelona:

Octaedro.

Puig, J.M.; Bär, B. (2016).

Reconocimiento y Aprendizaje-Servicio. RIDAS, Revista Iberoamericana de Aprendizaje y Servicio, 2, 139-165.

Trilla, J.; Novella, A. (2001). Educación y participación social de la infancia. Revista Iberoamericana de Educación, 26, 37-164. Disponible en http://www.rieoei.org/rie26a07.PDF

Rubio, L; Escardíbul, S. (2010). Todos somos meninos. En: Martín, X.; Rubio, L. Prácticas de Ciudadanía, (pp. 157171). Barcelona: Octaedro.

Uruñuela, P. (2016). Aprender cambiando el mundo. Madrid: Proyecto Agrupación ApS.

Xifra, C.; Escardíbul, S. (2018). La consolidación del aprendizaje-servicio en Fundesplai y en la educación en el tiempo libre. RIDAS, Revista Iberoamericana de Aprendizaje Servicio, 6, 115-126. 